\title{
Awakenings from persistent vegetative state: report of three cases with parkinsonism and brain stem lesions on MRI
}

\author{
W Matsuda, A Matsumura, Y Komatsu, K Yanaka, T Nose
}

J Neurol Neurosurg Psychiatry 2003;74:1571-1573

Three patients with a persistent vegetative state after severe head injury are reported. They recovered from a prolonged disturbance of consciousness after the administration of levodopa. These patients all had parkinsonian features. On magnetic resonance imaging, the distribution of lesions implied a diffuse axonal injury involving the substantia nigra or ventral tegmental area. The existence of patients whose dopaminergic systems may have been selectively damaged by a severe head injury should be recognised because such individuals may respond to levodopa treatment.

$\mathrm{P}$ ersistent vegetative state after severe head injury is a source not only of medical but also of legal and ethical debate, ${ }^{1}$ and there are occasional reports that have documented rapid recovery from this state. ${ }^{2}$ We present three patients, including one previously reported case (case $3),{ }^{4}$ all of whom showed remarkable neurological improvement after the administration of levodopa after three, seven, and 12 months in a persistent vegetative state after severe head injury. We discuss the relations among the effects of levodopa, post-traumatic parkinsonism underlying the persistent vegetative state, and the neuroradiological findings in these cases.

\section{CASE REPORT}

\section{Case 1}

A 14 year old boy was transferred to our hospital just after a traffic accident. He was comatose on arrival (Glasgow coma scale (GCS), 4). Computed tomography (CT) of the head showed a massive left subdural haematoma. The haematoma was removed and decompression craniotomy undertaken. Three months after the trauma, the patient was able to breathe without mechanical support through a tracheostomy. His cardiovascular, respiratory, renal, and gastrointestinal functions were sound, but he was doubly incontinent. He sometimes seemed to be awake, although his eyes could not follow moving objects and he could not obey simple verbal commands. Further examination by an experienced neurologist revealed predominant left sided rigidity/spasticity and tremor-like involuntary movements. Magnetic resonance imaging (MRI) at this stage showed the presence of high intensity lesions in the left dorsolateral midbrain (fig lA).

The clinical features of a persistent vegetative state with unusual findings in the limbs-that is, asymmetrical rigidity/ spasticity and involuntary tremor-implied post-traumatic parkinsonism. Levodopa treatment was recommended to relieve the patient's hypertonicity and involuntary movements. The administration of levodopa/benserazide (100/ $25 \mathrm{mg}$ twice a day) was begun through a gastric tube. Nine days later, the patient's involuntary movements were reduced and his eyes began to turn towards voices. Twenty days after the start of levodopa treatment, his rigidity/spasticity was reduced and he was able to obey simple verbal commands.
Three months after the start of treatment, he was able to walk while supporting himself with parallel bars, and had obtained the equivalent intelligence of an elementary school student. One year after the trauma, levodopa treatment was discontinued, and he was able to walk to high school by himself.

\section{Case 2}

A 27 year old man was involved in a traffic accident and was transferred to another hospital. He was comatose on arrival (GCS 4). CT showed a right epidural haematoma and traumatic subarachnoid haemorrhage with diffuse swelling. He underwent haematoma evacuation and internal decompression (right occipital lobectomy). Three months after the trauma, he was transferred to our hospital. He had a tracheostomy tube and a gastric tube inserted. His general state was stable, but he was doubly incontinent. He sometimes seemed to be awake, although his eyes could not follow moving objects and he could not obey simple verbal commands. MRI at this stage revealed the presence of high intensity lesions in the right cerebral peduncle and the left dorsolateral midbrain (fig 1B). Six months after the trauma, a deep brain stimulation system was implanted in the left thalamus, but no neurological improvement was observed. One year after the trauma, there was no improvement.

The patient was diagnosed as being in a persistent vegetative state, but a further examination by an experienced neurologist revealed predominantly right sided rigidity.

Administration of levodopa (450 mg a day) was begun through the gastric tube. Eight days later, the patient's rigidity was reduced and his eyes began to follow moving objects. Twenty five days after the start of the treatment, he was transferred to a rehabilitation centre, and the levodopa prescription was changed to levodopa/benserazide (100/ $25 \mathrm{mg}$ three times a day). He began to respond to his family and the nurses by blinking his eyes. He improved further to be able to express "yes" or "no" by blinking, and thereafter by operating a simple electronic signal with his right hand. Ten months after the start of treatment, he began to use a word processor to express his thoughts. One year after the start of the therapy, he was extubated; he said, "I want to eat sushi and drink beer." In this patient, the levodopa treatment is to be continued indefinitely.

\section{Case 3}

A 51 year old man was transferred to our hospital just after a traffic accident. He was comatose on arrival (GCS 6) and had a left hemiparesis with a left oculomotor nerve palsy. CT examination showed a traumatic subarachnoid haemorrhage without a mass lesion. MRI showed high intensity lesions in the right cerebral peduncle and the left dorsolateral midbrain (fig lC). From three months after the trauma, the patient received thyrotropin releasing hormone tartrate intravenously at a dose of $2 \mathrm{mg}$ a day for 10 days each month for three cycles, but no effect was observed. Seven months after the trauma, he had a tracheostomy tube and a gastric tube inserted. His general state was stable, but he was doubly 

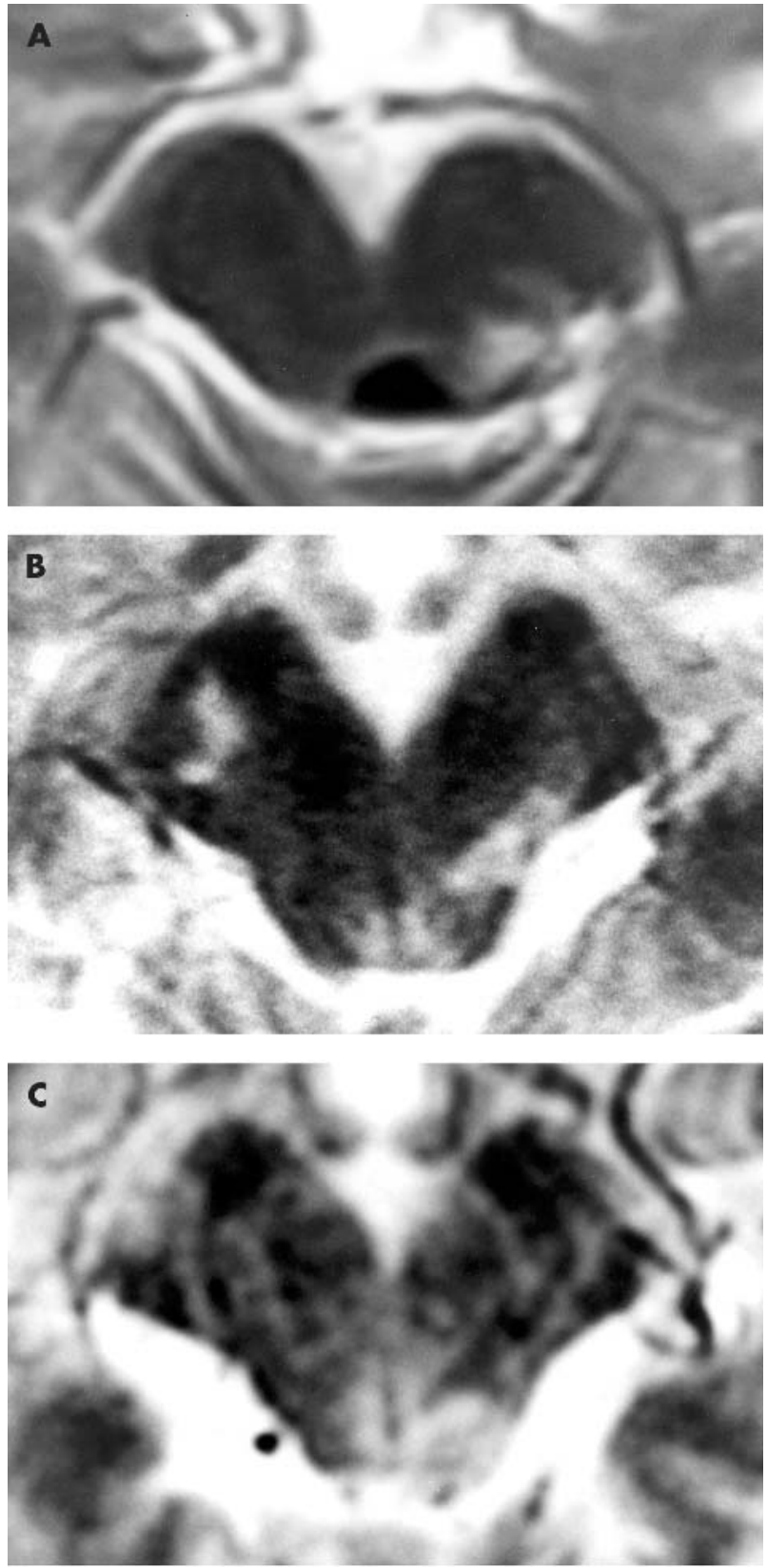

Figure 1 (A) High intensity lesions in the left dorsolateral midbrain on T2 weighted magnetic resonance imaging in case 1. (B) High intensity lesions in the right cerebral peduncle and the left dorsolateral midbrain in case 2. (C) High intensity lesions in the right cerebral peduncle and the left dorsolateral midbrain in case 3.

incontinent. He could not obey simple verbal commands and was diagnosed as being in a persistent vegetative state. A further examination by an experienced neurologist revealed akinesia and predominantly right sided rigidity/ spasticity of his extremities.

Administration of levodopa/carbidopa (100/10 mg three times a day) was begun through the gastric tube. Four days later, the patient's akinesia and rigidity were reduced and he was able to obey simple verbal commands. Two months after the start of the treatment, he was extubated and was able to say his name and address correctly. Six months after the start of treatment, he was transferred to another hospital to continue rehabilitation. In this patient, levodopa is to be continued indefinitely.

\section{DISCUSSION}

All these cases were deeply comatose after severe head injury without a period of lucidity. The patients remained unresponsive to simple verbal commands, and their condition was unchanged for three to 12 months, despite intensive treatment including surgery. All the patients were diagnosed as being in a persistent vegetative state at three, seven, and 12 months after the trauma, respectively.

For adult patients in a vegetative state after traumatic brain injury, the prognosis for recovery remains poor. ${ }^{5}$ Data from the task force report ${ }^{6}$ in 1994 showed recovery in 33\% of adult patients in a vegetative state for three months after a severe head injury, $13 \%$ after three to six months, and $6 \%$ after six to 12 months. Thus the rapid recovery after the start of levodopa treatment in the present cases suggests a significant relation to that treatment, particularly in cases 2 and 3, who were in a vegetative state for seven and 12 months, respectively.

These three patients were diagnosed as having brain stem injury on MRI. In the previous reports, ${ }^{7-9}$ recovery from brain stem injury is dependent on the extent of the injury. High intensity lesions on T2 weighted MRI of the cerebral peduncle or dorsolateral midbrain were highly significant predictors of non-recovery. ${ }^{7}$ Ipsilateral brain stem damage usually has a favourable prognosis but there is poor recovery from bilateral damage, despite similar initial GCS scores. ${ }^{8}$ Another report of acute stage MRI appearances suggested that deep dorsal lesions were also an indicator of poor recovery. ${ }^{9}$ Thus the patients reported here had been predicted to have little chance of recovery, but actually showed remarkable improvement.

A careful neurological evaluation of these three cases revealed asymmetrical rigidity, akinesia, or tremor, implying parkinsonism. These clinical features are unusual findings in persistent vegetative state. Previous reports of the MRI findings in patients with post-traumatic parkinsonism have described lesions involving the substantia nigra or pallidum, ${ }^{10}{ }^{11}$ or the caudate and lenticular nuclei..$^{12}$ The presence of post-traumatic parkinsonism and the probable involvement of the substantia nigra and ventral tegmental areasuggested by the MRI findings-implied a diffuse axonal injury involving the dopaminergic pathway in these patients. Injury to these areas may cause defects in the nigrostriatal system (A9), the mesocortical system, or the mesolimbic system (A10). Thus we considered that these patients were in a persistent vegetative state associated with post-traumatic parkinsonism. Primary brain stem injury involving selective dopaminergic systems may have modified their neurological status. Severe rigidity and akinesia would also have locked them into a bedridden state. Although there was no response to the levodopa treatment in a previously reported case of post-traumatic Parkinson's syndrome, ${ }^{12}$ presynaptic damage in the dopaminergic systems may explain the remarkable effects of levodopa in the present cases. There have been previous reports of clinical improvement from post-traumatic persistent vegetative state with the administration of levodopa, ${ }^{23}$ but the possibility of benefit from the dopaminergic treatment was considered equivocal.

\section{Conclusions}

Levodopa treatment should be considered for patients in a persistent vegetative state with atypical features in their limbs that are suggestive of extrapyramidal dysfunction and who have MRI evidence of lesions in the dopaminergic pathway, particularly presynaptic lesions in areas such as the substantia nigra or ventral tegmentum. These cases emphasise the possibility of treating some patients who are in a persistent vegetative state and who would otherwise remain bedridden for the rest of their lives. 


\section{Authors' affiliations}

W Matsuda, Y Komatsu, Department of Neurosurgery, Tsukuba Medical Centre Hospital, Ibaraki, Japan

A Matsumura, K Yanaka, T Nose, Department of Neurosurgery, Institute of Clinical Medicine, University of Tsukuba, Ibaraki, Japan

Competing interests: none declared

Correspondence to: Dr Wakoto Matsuda, Department of Neurosurgery, Tsukuba Medical Centre Hospital, 1-3-1 Amakubo, Tsukuba, lbaraki 305-0005, Japan; wako@mua.biglobe.ne.jp

Received 17 February 2003

In revised form 16 April 2003

Accepted 25 April 2003

\section{REFERENCES}

1 Executive Board, American Academy of Neurology. Position of the American Academy of Neurology on certain aspects of the care and management of the persistent vegetative state patient. Neurology 1989:39:125-6.

2 Haig AJ, Ruess JM. Recovery from vegetative state of six months' duration associated with Sinemet (levodopa/carbidopa). Arch Phys Med Rehabil 1990:71:1081-3.
3 Higashi K, Hatano M, Abiko S, et al. Clinical analysis of patients recovered from persistent vegetative state, with special emphasis on the therapeutic and prophylactic effects of L-dopa. Brain Nerve 1978;30:27-35, [in Japanese].

4 Matsuda W, Sugimoto K, Sato N, et al. A case of primary brain-stem injury recovered from persistent vegetative state after L-dopa administration. Brain Nerve 1999;51:1071-4, [in Japanese]

5 Zeman A. Persistent vegetative state. Lancet 1997;350:795-9.

6 Task Force. Multi-society task force on PVS. Medical aspects of the persistent vegetative state. N Engl J Med 1994;330:1572-9.

7 Kampfl A, Schmutzhard E, Franz G, et al. Prediction of recovery from posttraumatic vegetative state with cerebral magnetic-resonance imaging. Lancet 1998;351:1763-7.

8 Matsumura A, Mitsui I, Ayuzawa S, et al. Prediction of reversibility of the brain stem dysfunction in head injury patients. In: Nakamura N, ed. Recent advances in neurotraumatology. Tokyo: Springer-Verlag, 1993:192-5.

9 Shibata $Y$, Matsumura A, Meguro K, et al. Differentiation of mechanism and prognosis of traumatic brain stem lesions detected by magnetic resonance imaging in the acute stage. Clin Neurol Neurosurg 2000;102:124-8.

10 Krauss JK, Wakhloo AK, Nobbe F, et al. Lesions of dentatothalamic pathways in severe post-traumatic tremor. Neurol Res 1995;17:409-16.

11 Koeda T, Takeshita K. A case report of remarkable improvement of motor disturbances with L-dopa in a patient with post-diffuse axonal injury. Brain Dev 1998;20:124-6.

12 Doder M, Jahanshahi M, Turjanski N, et al. Parkinson's syndrome after closed head injury: a single case report. J Neurol Neurosurg Psychiatry 1999;66:380-5.

\section{NEURONLINE}

\section{Eye simulator: http://cim.ucdavis.edu/EyeRelease}

- yeSim is a well designed, interac-

- tive website that simulates eye movements and pupillary responses. Virtual lesions induce defects that are not subtle, making the simulator more useful for teaching undergraduates and junior postgraduates than for simulating "the real thing". The website is readily accessible and the explanations are clear. Two multimedia application programmes are required to run EyeSim; these can be easily downloaded from the opening page of the website.

Although there is a demonstration mode that (literally) talks the user through a very simple examination of ocular motility and demonstrates third, fourth, and sixth nerve palsies, one of the most pleasing features of the website is one's ability to interact with it. The website presents a virtual patient whose two eyes follow the computer mouse. One can click on a menu to disable one or more extra ocular muscles and observe the resulting eye movements. The eye movement abnormalities are severe but accurate for acute impairment. Although there are conjunctival blood vessels that might have been used as reference points, torsional movements are, unfortunately, not simulated.

The pupil responses can be assessed with a virtual pen torch. There is a useful simulation of the relative afferent pupillary defect, RAPD (relative afferent pupillary defect; which the authors term a 'mild RAPD'), although there is no pupillary escape that can make assessing for a RAPD in 'real life' difficult. However, what they term a marked RAPD is a total APD (there is no direct pupil response to light).

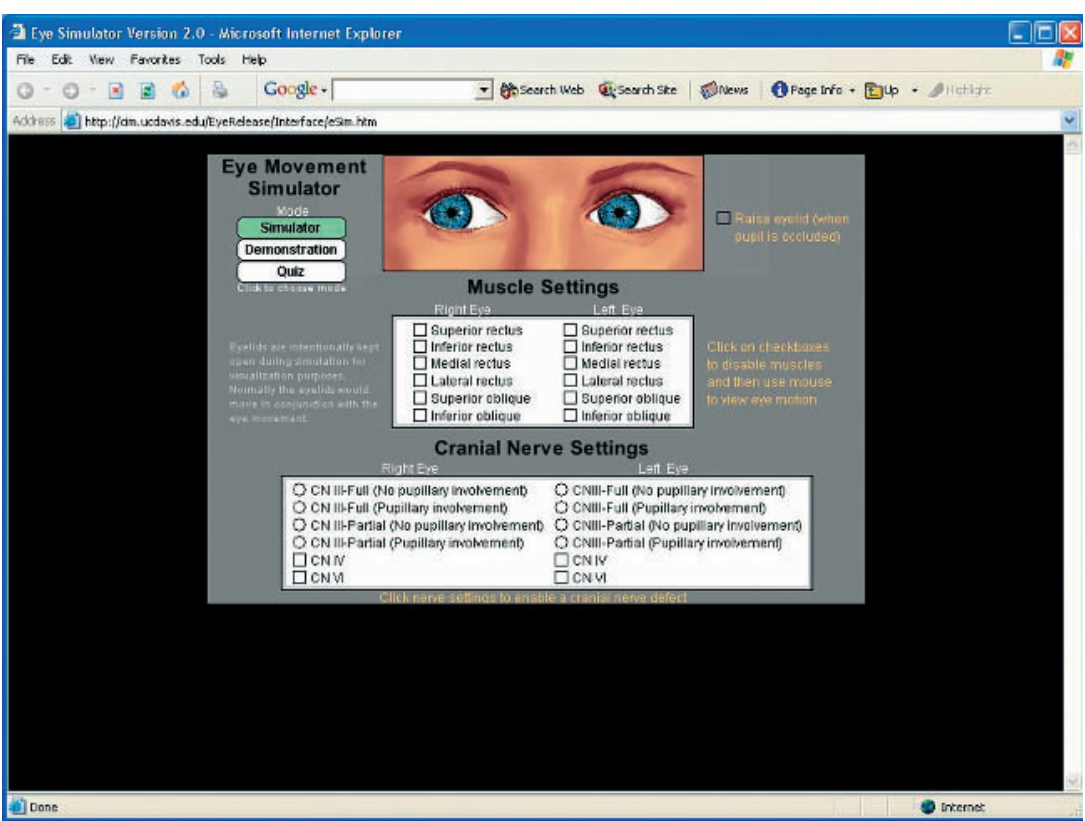

The terminology used in the discussion of third nerve palsies will not be to every user's taste. The authors use full or partial to refer to the degree of impairment of the affected muscles rather than whether only certain muscle groups are affected. Pupil involvement is treated separately.

The simulation and discussion of fourth nerve palsies is simplified. Mention could have been made of anomalous head position and that the defect in primary position is dependent on which eye is fixing.

There are three straightforward interactive clinical cases. We have some reservations about the proposed management of case 1 (in question 6) and the discussion of case 3. There appears to be a missing pupil defect in case 2 . But you will have to visit the site yourself to see if you agree..

R M Bracewell Centre for Cognitive Neuroscience, School of Psychology, University of Wales, Bangor, Gwynedd LL57 2AS; Walton Centre for Neurology and Neurosurgery, Lower Lane, Fazakerley, Liverpool L9 7ப, UK

I R C Anderson

Department of Ophthalmology, Mater Health Services, Raymond Terrace, South Brisbane, Qld, Australia 4101, Australia

Correspondence to: R M Bracewell; m.bracewell@bangor.ac.uk 